\title{
Physics Prospects from Accelerated Radioactive Ions at CERN
}

\author{
P. A. Butler*a and the ISOLDE collaboration
}

aSOLDE, CERN, CH-1211 Geneva 23, Switzerland, http://isolde.cern.ch,

Through the advent of post-accelerated beams with REX-ISOLDE at CERN, probing nuclear properties using transfer reactions and Coulomb excitation of exotic nuclear species is now possible. REX ISOLDE currently provides beams of energy $2.2 \mathrm{MeV} / \mathrm{u}$ (soon be upgraded to $3.1 \mathrm{MeV} / \mathrm{u}$ ) into the gamma-ray MINIBALL array, and other instrumentation, at the secondary target position. Examples of research topics currently addressed using REX are presented.

Scheduled energy up-grades will increase the physics potential even further. The goal for the next five years will be to accelerate ions up to $5 \mathrm{MeV} / \mathrm{A}$ and higher energies. Increase of primary beam intensity will also be achieved in a phased approach, with a significant enhancement provided by the proposed Superconducting Proton Linac as the primary accelerator.

\section{Introduction}

ISOLDE is a collaboration that contains members from about 80 institutions worldwide. The experimental programme consists of 35-40 active experiments that make use of 5-10 independent spectrometers and other complex instruments in a typical year. The lowenergy radioactive beams of exotic nuclei available at ISOLDE, unrivalled for many nuclear species in intensity, purity and beam quality, are crucial vehicles to understand the details of the nuclear many-body system at the extremes as well as increasing our knowledge about nuclear astrophysics and fundamental interactions and being an important tool for research in condensed matter and life sciences.

The experiments carried out at ISOLDE are diverse and state-of-the-art. In addition to a large range of detectors of different types for decay and in-beam experiments, there are instruments for laser spectroscopy and nuclear orientation of exotic nuclei, permitting studies of nuclear ground state properties far from stability. The triple-trap ISOLTRAP spectrometer can routinely achieve accuracies of $10^{-8}$ in mass measurements of unstable nuclei, and is complemented by the MISTRAL spectrometer that is able to measure masses of the most short-lived species such as ${ }^{11} \mathrm{Li}$. In the last two years the Radioactive beam EXperiment REX-ISOLDE has been operational and is used to test new concepts of post acceleration of radioactive ions as well as use the accelerated beams for experiments in nuclear physics, astrophysics and solid state physics.

For a comprehensive review of activities at ISOLDE see [1]

*permanent address: Oliver Lodge Laboratory, University of Liverpool, Liverpool L69 7ZE 


\section{REX-ISOLDE}

REX [2] makes use of the large variety of radionuclides that have been extracted from the on-line mass separator ISOLDE. In order to use these beams significant developments in beam cooling and charge breeding were required, as whereas the output of the ISOLDE primary mass separator is a quasi-DC beam (the intensity varies between the $1.2 \mathrm{~s}$ primary proton pulses according to the release characteristics and the nuclide half-life) having charge state $1^{+}$, the low duty-factor post accelerator requires multiply-charged $(\mathrm{A} / \mathrm{q}<$ 4.5) pulsed beams.

The first stage of the preparation of the ISOLDE beam is to continuously inject the beam from the mass separator into REXTRAP [3] where it is cooled, accumulated and extracted as bunches. The accumulation and cooling is achieved by collisions with a buffer gas. In the collisions the loss of energy of the ion enables it to be trapped within a potential barrier. The barrier is reduced every $20 \mathrm{~ms}$ so that the accumulated ions are ejected as a $20 \mu \mathrm{s}$ pulse. The transverse emittance of the beam is also reduced by the cooling procedure. This is important because the next stage, REXEBIS [4](REX Electron Beam Ion Source), has a small acceptance (3 $\mathrm{mm} \mathrm{mrad})$. REXEBIS employs a $500 \mathrm{~mA} 5$ $\mathrm{keV}$ electron beam that is focussed to a high current density $\left(250 \mathrm{~A} / \mathrm{cm}^{2}\right)$ by a $2 \mathrm{~T}$ axial magnetic field. The ion beam is confined longitudinally by applied electric potentials and undergoes stepwise ionization via electron collisions until its mean charge state has the desired value. For $\mathrm{A}<50$ the breeding time in REXTRAP to reach $\mathrm{A} / \mathrm{q}=3-4$ is around $20 \mathrm{~ms}$. For ${ }^{133} \mathrm{Cs}$ ions the breeding time was $80 \mathrm{~ms}$.

The yield of the radioactive isotopes from ISOLDE can be several orders of magnitude lower than the amount of residual gas ions from $\mathrm{C}, \mathrm{N}, \mathrm{O}$ and $\mathrm{Ar}$ coming out of the EBIS. Therefore, a mass separator is employed with a q/A-resolution of about 1/150 which is sufficient to select the highly charged rare radioactive ions from rest-gas contaminants.

In the first stage of the REX-ISOLDE linac the ions are accelerated from $5 \mathrm{keV} / \mathrm{u}$ to $300 \mathrm{keV} / \mathrm{u}$ by a 4-rod RFQ. In order to match the beam from the RFQ into the acceptance of the IH (Interdigital-H-type)-structure a section consisting of two magnetic quadrupole triplet lenses and a rebuncher is required. A feature of the REX-ISOLDE-IH resonator is the possibility to vary the final energy between 1.1 and $1.2 \mathrm{MeV} / \mathrm{u}$ by adjusting the gap voltage distribution via two capacitive plungers and by adjusting the rf-power fed into the resonator. The lower final energy of the IH-structure is important for deceleration of the ions down to $0.8 \mathrm{MeV} / \mathrm{u}$, since the deceleration from $1.2 \mathrm{MeV} / \mathrm{u}$ down to $0.8 \mathrm{MeV} / \mathrm{u}$ through the 7 -gap resonators would perform a non-acceptable phase spread at the target.

The high-energy section $(0.8-2.2 \mathrm{MeV} / \mathrm{u})$ of the linac consists of three 7 -gap resonators. These spiral resonators are designed and optimised for synchronous velocities of $\beta=5.4 \%$, $6.0 \%$ and $6.6 \%$. Each resonator has a single resonance structure, which consists of a copper half shell and three arms attached to both sides of the shell. All linac structures are operated at $101.28 \mathrm{MHz}$.

In the summer of 2003 an additional 9-gap IH structure, operating at a frequency of 202.56 MHz will be installed in the high-energy section of the linac. This will provided ions accelerated to $3.1 \mathrm{MeV} / \mathrm{u}$. 


\section{Physics Programme of REX}

Through the advent of post-accelerated beams with REX-ISOLDE, probing nuclear properties using transfer reactions and Coulomb excitation of exotic nuclear species is now possible. REX ISOLDE currently provides accelerated beams to two secondary target positions, including the HPGe gamma spectrometer [5] which in its present phase of operation has a a full-energy peak efficiency of $\approx 10 \%$ for a photon energy of $1 \mathrm{MeV}$. REX is operational and has already accelerated several species of radioactive ions, e.g. 10,000 ions/s of ${ }^{29} \mathrm{Na}$; it has the capability to accelerate mass 130 ions and heavier with efficiency of a few percent of the ion source yield. In 2003 REX-ISOLDE was integrated into the standard operation of CERN facilities.

In the following two sub-sections examples are given of the current research programme in nuclear physics that uses REX.

\subsection{Halo Nuclei}

Two-neutron halo states are situated at the threshold that separates the discrete and continuum spectra. Precise information about the continuum states are therefore important for an understanding of the halo structure. One of the first experiments [6] using REX-ISOLDE was to investigate scattering states in ${ }^{10} \mathrm{Li}$ using the $\mathrm{d}\left({ }^{9} \mathrm{Li},{ }^{10} \mathrm{Li}\right) \mathrm{p}$ reaction at $2.36 \mathrm{MeV} / \mathrm{u}$, in particular the $1 s_{\frac{1}{2}}$ state which should lie very low in the ${ }^{10} \mathrm{Li}$ spectrum. This experiment employed a $64 \mu \mathrm{m}$ segmented (16 x 16) Si $\Delta$ E detector backed by a $1 \mathrm{~mm}$ Si detector to provide particle identification. Preliminary analysis of these data suggest that the lowest unbound states in ${ }^{10} \mathrm{Li}$ can be identified but further experiments at a higher bombarding energy are necessary.

\subsection{Collective Properties of Very Neutron Rich Nuclei}

One of the most interesting topics in nuclear structure research far from stability is the behaviour of nuclei with $\mathrm{Z} \approx 12$ and $\mathrm{N} \approx 20$, where under the extreme conditions of large $\mathrm{N}$-Z-asymmetry and weak binding, the filling of the $\mathrm{f}_{\frac{7}{2}}$ intruder orbits causes an inversion of the standard shell ordering and hence the breakdown of the $\mathrm{N}=20$ shell closure.

The vanishing of the $\mathrm{N}=20$ shell closure is illustrated in Fig. 1, where the energies of the first $2^{+}$states for the $\mathrm{Mg}$ and $\mathrm{Si}$ isotopes are shown as a function of neutron number. The high energy for the $\mathrm{N}=20$ nucleus ${ }^{34} \mathrm{Si}$ shows a strong shell effect at $\mathrm{Z}=$ 14 , while for ${ }^{32} \mathrm{Mg}$ the energy drops below that of ${ }^{30} \mathrm{Mg}$ indicating a complete vanishing of the shell closure at $\mathrm{Z}=12$. The $\mathrm{B}\left(\mathrm{E} 2 ; 0_{g} \rightarrow 2_{1}\right)$ values for the $\mathrm{Mg}$ isotopes also demonstrate no indication of a shell closure at $\mathrm{N}=20$ for $\mathrm{Z}=12$. There is, however, a rather large uncertainty on the measured values and for ${ }^{30,32} \mathrm{Mg}$ different groups employing the same experimental method (intermediate-energy Coulomb excitation) have obtained different results. This might indicate that systematic uncertainties are present in these measurements, such as the presence of Coulomb-nuclear interference, or the effect of feeding from the many higher-lying states that are excited in the single-step process.

One of the first experiments [7] employed $2.2 \mathrm{MeV} / \mathrm{u}^{30} \mathrm{Mg}$ beams that are Coulomb

excited by $1 \mathrm{mg} / \mathrm{cm}^{2} \mathrm{Ni}$ foils; the $\gamma$-radiation was observed using MINIBALL consisting of 8 3-module, 6 -fold segmented HPGe detectors. The beam intensity was $\approx 10^{4}$ ions per second for a total irradiation time of 15 hours. Figure 2 shows the $\gamma$-ray spectrum recorded in coincidence with an annular, segmented silicon detector (CD detector) that 


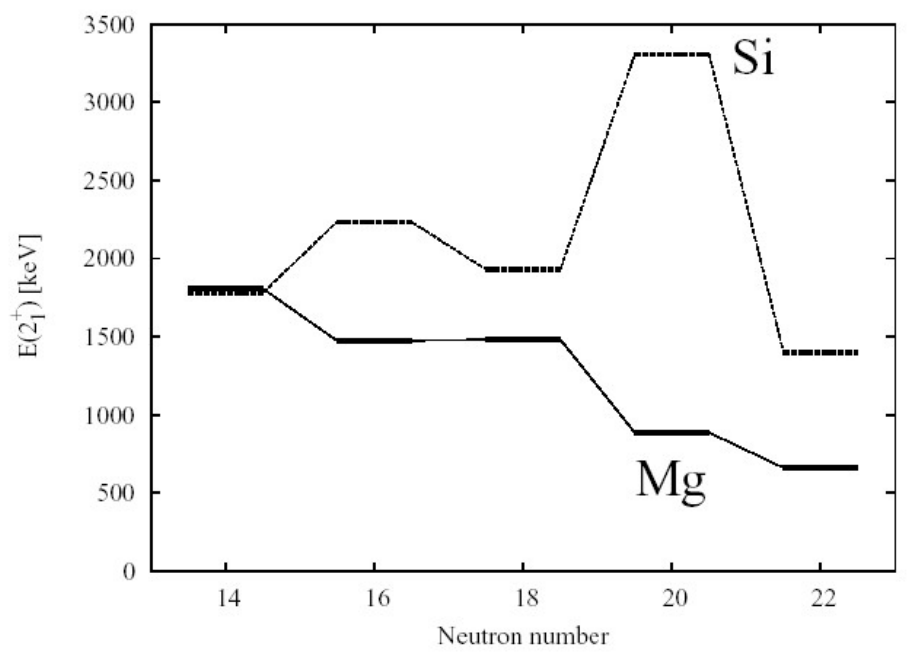

Figure 1. Energies of the first $2^{+}$states for the $\mathrm{Mg}$ and $\mathrm{Si}$ isotopes [7]

detected forward scattered beam particles. The $2^{+} \rightarrow 0^{+}$transition in ${ }^{30} \mathrm{Mg}$ can clearly be identified. Further experiments are in progress to improve statistics and measure the $\mathrm{B}(\mathrm{E} 2)$ value of this transition.

\section{Future Upgrades of ISOLDE and REX}

Scheduled energy and intensity up-grades will increase the physics potential of ISOLDE even further. The goal for the next five years will be to accelerate ions to $4.3 \mathrm{MeV} / \mathrm{u}$ and beyond, with an increase of primary beam intensity to $5 \mu \mathrm{A}$ or higher. This enhancement of the ISOLDE facility is called "High Intensity and Energy ISOLDE" or HIE-ISOLDE. The energy upgrade can be considered in two phases: (1) to increase the energy to 4.3 $\mathrm{MeV} / \mathrm{u}$ by 2005 that enables certain classes of nuclear reactions to be used on a wide range of nuclei and (2) further increases in energy that will enable all reactions to be employed for all nuclei. The ISOLDE hall will be extended in order to house the additional linac cavity resonators and other instrumentation such as new recoil spectrometers for detection of fusion products. Another new facility, REX*, will provide low energy radioactive ions from a charge breeder ion source either mounted on a high voltage platform or used in conjunction with a variable-energy RFQ. This will provide beams with continuously variable energy from $<100 \mathrm{keV}$ to $>10 \mathrm{MeV}$ for astrophysics and condensed matter studies. Figure 3 shows a scheme of HIE-ISOLDE and its applications. In parallel with the energy upgrade, the proton beam intensity on the primary target will be increased. In the next few years, the available beam intensity from the PS booster, through the provision of $\mathrm{H}^{-}$injected beam from a new primary injector linac (LINAC 4) and a faster cycling time in the PS Booster, will be increased three-fold, resulting in a significant increase in beam intensity available to ISOLDE. The development of primary target and ion-sources that can withstand these and higher beam intensities will be made in parallel 


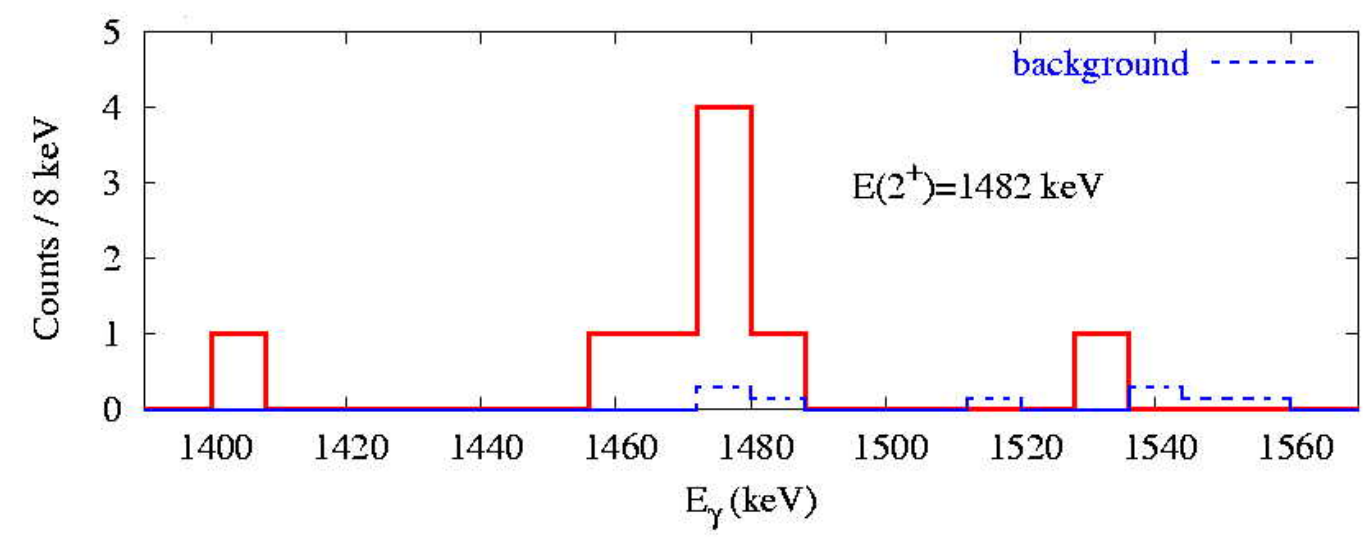

Figure 2. $\gamma$-ray spectrum following Coulomb excitation of ${ }^{30} \mathrm{Mg}$ ions [8]

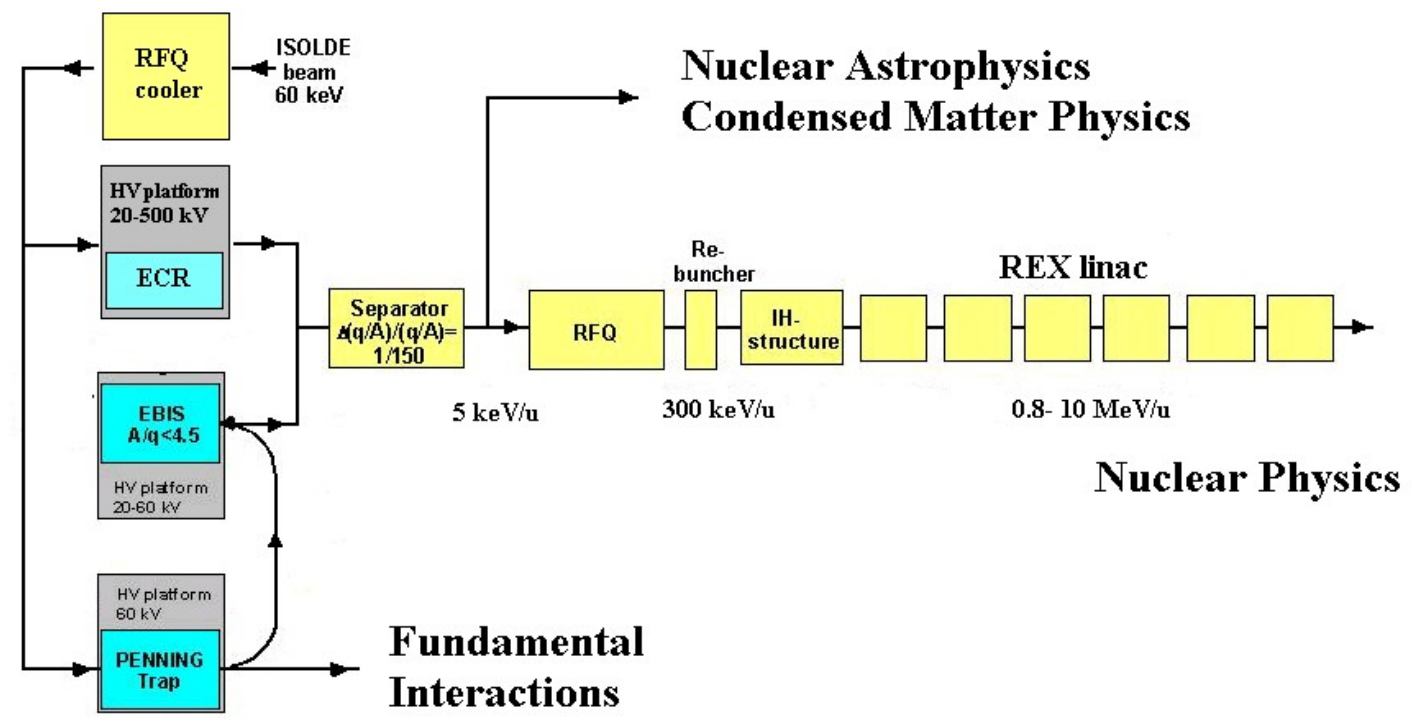

Figure 3. Scheme of HIE-ISOLDE and its applications 
with the intensity upgrade.

\section{The SPL Project}

CERN is currently considering the scientific case for a high power proton driver, the Superconducting Proton Linac [9]. This accelerator will provide $2.2 \mathrm{GeV}, 11 \mathrm{~mA} \mathrm{CW}$ proton beams for enhanced injection to the PS and thence to the LHC, and to provide intense proton beams for neutrino production and to a next generation radioactive ion beam (RIB) facility. There are a number of scenarios for neutrino production. In the Neutrino Factory the proton beam strikes a fixed target producing pions which are captured in a solenoidal channel where they decay into muons. The muons are subsequently cooled and accelerated before injection into a bow-tie or triangle shaped decay ring. A new concept for neutrino beams having strong synergies in accelerator and target technology with a RIB facility is the beta-beam [10] in which ${ }^{6} \mathrm{He}$ and ${ }^{18} \mathrm{Ne}$ radioactive ions are accelerated to a Lorentz gamma of $\approx 100$ before injection into a decay ring, thus producing a flavour-pure beam of anti-neutrinos and neutrinos respectively.

For the next generation RIB facility, the leading European nuclear physics facilities have prepared a preliminary design based on the Isotope Separation On Line approach, called EURISOL [11]. The high duty cycle of the CERN SPL (at least $150 \mu$ s available of the $2.8 \mathrm{~ms}$ pulse width, repeated at a frequency of $50 \mathrm{~Hz}$ ) provides a good match to the specification of the primary driver of EURISOL. This will give a lower peak intensity of the proton beam than the present PS-Booster ISOLDE facility (thereby increasing the target lifetime), while the average current for direct RIB production can be increased to $100 \mu \mathrm{A}$ or more compared to the present value of $\approx 2 \mu \mathrm{A}$. The higher proton energy will give an order of magnitude increase (over that from the existing ISOLDE facility) in the formation cross-sections for the light neutron rich target fragmentation products, while operating the SPL at lower energies than the nominal $2.2 \mathrm{GeV}$ permits the RIB production cross-section of the spallation products to be optimised. The high power available (4 MW) also allows the use of a spallation neutron target for production of intense beams of fission fragments in a secondary target.

The driver beam will be transported to both the existing ISOLDE facility and a new target area that is located underground. Post-accelerated RIB beams of energies up to and beyond $10 \mathrm{MeV} / \mathrm{u}$ will be transported to at least three experimental areas, with possibilities to be explored of injection into the PS Booster to accelerate heavy radioactive ions to $100 \mathrm{MeV} / \mathrm{u}$ or to inject into storage rings with electrons, muons, or antiprotons.

\section{Outlook}

ISOLDE at CERN is currently undergoing a renaissance in terms of its application to studies in nuclear, astro-, and condensed matter physics, thanks to the advent of the post-accelerator REX. The planned upgrades in terms of beam energy and intensity, first HIE-ISOLDE later this decade and then SPL-ISOLDE from 2010, will ensure that ISOLDE remains a leading centre world-wide for research using accelerated radioactive ions. 
I am grateful to U. Bergmann, O. Niedermaier, and H. Scheit for providing preliminary data, and to M. Lindroos and T. Nilsson for helpful discussions.

\section{REFERENCES}

1. D. Forkel-Wirth, G. Bollen (eds), Hyperfine Interactions, volume 129, 2000

2. D. Habs et al., Hyperfine Interactions, 129 (2000) 43

3. G. Bollen et al., Nucl. Instrum. Meth. A, 368 (1996) 675

4. B.H. Wolf et al., Nucl. Instrum. Meth. B, 204 (2003) 428

5. J. Eberth et al., Prog. Part. Nucl. Phys. 46 (2001) 389

6. B. Jonson et al.: Study of the unbound nuclei ${ }^{10} \mathrm{Li}$ and ${ }^{7} \mathrm{He}$ at REX ISOLDE CERNISC-98-11 ; ISC-P-100, 1998

7. H. Scheit et al.: Evolution of single particle and collective properties in the neutronrich Mg isotopes CERN-INTC-2002-020 ; INTC-P-159, 2002

8. H. Scheit and O. Niedermaier, priv. comm.

9. B. Autin et al.: Conceptual design of the SPL, a high-power superconduction $\mathrm{H}^{-}$ Linac at CERN, CERN-2000-012, 2000

10. P. Zucchelli, Phys. Lett. B, 532 (2002) 166

11. J. Vervier et al., http://www.ganil.fr/eurisol 